\title{
Quantization improves stabilization of dynamical systems with delayed feedback
}

\author{
Gabor Stepan, ${ }^{1}$ John G. Milton, ${ }^{2}$ and Tamas Insperger ${ }^{3}$ \\ ${ }^{1}$ Department of Applied Mechanics, Budapest University of Technology and Economics, 1111 Budapest, \\ Hungary \\ ${ }^{2}$ W. M. Keck Science Center, The Claremont Colleges, Claremont, California 91711, USA \\ ${ }^{3}$ Department of Applied Mechanics, Budapest University of Technology and Economics and MTA-BME \\ Lendület Human Balancing Research Group, 1111 Budapest, Hungary
}

(Received 3 May 2017; accepted 3 August 2017; published online 12 October 2017)

\begin{abstract}
We show that an unstable scalar dynamical system with time-delayed feedback can be stabilized by quantizing the feedback. The discrete time model corresponds to a previously unrecognized case of the microchaotic map in which the fixed point is both locally and globally repelling. In the continuous-time model, stabilization by quantization is possible when the fixed point in the absence of feedback is an unstable node, and in the presence of feedback, it is an unstable focus (spiral). The results are illustrated with numerical simulation of the unstable Hayes equation. The solutions of the quantized Hayes equation take the form of oscillations in which the amplitude is a function of the size of the quantization step. If the quantization step is sufficiently small, the amplitude of the oscillations can be small enough to practically approximate the dynamics around a stable fixed point. Published by AIP Publishing. https://doi.org/10.1063/1.5006777
\end{abstract}

Digital feedback controllers are having an increasing impact on human activities. Examples range from the control of single-atom trajectories ${ }^{1}$ to the development of brain-device interfaces, ${ }^{2}$ from the treatment of human diseases using closed-loop drug delivery systems, ${ }^{3}$ to the design of driverless automobiles. ${ }^{4}$ An important component of these controllers is an analog-to-digital (A/D) conversion by which a continuous (or analog) signal is converted into a series of numbers proportional to the signal. This $\mathrm{A} / \mathrm{D}$ conversion is essential to enable the digital microprocessors to track the controlled variable: the reverse D/A conversion makes it possible for the microprocessor to affect control. The effect of $A / D$ and $D / A$ conversion is to introduce a quantization into both the time domain ("sampling") and the controlling force ("round off"). Although it is well known that these quantizations can introduce spurious oscillations ${ }^{5,6}$ and small amplitude micro-chaotic fluctuations into the control dynamics, ${ }^{7,8}$ less is known about the possible benefits of feedback quantization for control. Here, we analyze a generic scalar model for feedback control and show that a "coarse-grained" signal quantization can contribute to the stabilization of unstable dynamical systems in the presence of feedback delays.

\section{INTRODUCTION}

The advantages of digital feedback control over continuous, or analog, control are well documented. ${ }^{9,10}$ Digital controllers are cheaper, easier to configure, more adaptable, and less prone to the effects of environmental fluctuations. However, the dynamics which arise in the setting of digital control can sometimes be counter-intuitive. Examples include the appearance of an oscillation whose frequency is within a range far smaller than the sampling frequency and the appearance of low amplitude stochastic-like fluctuations in the desired motion, referred to as microchaos. ${ }^{7,8}$ Even more paradoxical is the prediction that transiently stable solutions can occur for parameter ranges that for analog feedback would be unstable. ${ }^{11,12}$ These quantization effects may not be unique to man-made devices. The conversion of sensory inputs into a train of discrete action potentials underlies sensory-motor encoding in the nervous system. Quantization of voluntary movements is manifested in the visually guided movements in infants ${ }^{13}$ and in patients with brain injury. ${ }^{14}$ Moreover, sensory dead zones, namely, a range of inputs which give the same output, arise both in neural control ${ }^{15}$ and in A/D conversion $^{5,6}$ and result in the appearance of limit cycle oscillations.

This communication focuses on the observation that certain unstable time-delayed dynamical systems can be practically stabilized by round off. Our discussion is organized as follows. In Sec. II, we review the effects of feedback digitization on the dynamics of time delayed feedback control from the perspective of a discrete time map referred to as the microchaos map. Our focus is on the stable dynamics which arise when the fixed-point is locally unstable (repelling). Section III demonstrates that the stable dynamics that arise when this fixed point is globally attracting include limit cycle oscillations and microchaos. Surprisingly, stable and metastable oscillatory dynamics can also arise if this fixed point in the presence of continuous feedback is, in addition, globally repelling. Section IV shows an application of these observations for stabilizing the Hayes equation ${ }^{16}$ for delayed feedback control using quantization. Since the amplitude of the fluctuations is proportional to the size of the quantization step, it becomes possible to replace the dynamics around an unstable fixed point with stable, low amplitude fluctuations. This result may be sufficient for many practical applications. 


\section{DIGITAL FEEDBACK}

Consider the dynamics of an over-damped unstable system described by

$$
\dot{x}(t)=q x(t)-f(x(t)),
$$

where $x$ is a state variable, $q>0$ is a constant, and $f$ describes the feedback. There are two effects of a digital implementation of (1). First, the fact that $f$ is determined from the sampled values of $x$ introduces a temporal piecewise smooth dynamics ${ }^{7,8}$ governed by

$$
\dot{x}(t)=q x(t)-f\left(x\left(t_{j}\right)\right), \quad t \in\left[t_{j}, t_{j+1}\right),
$$

where $t_{j}=j \Delta t, j=0,1,2, \ldots$ are the sampling instants and $\Delta t$ is the constant sampling time. Temporal sampling introduces a delay in the system, hence Eq. (2) can also be written as

$$
\dot{x}(t)=q x(t)-f(x(t-\rho(t))),
$$

where

$$
\rho(t)=t-\Delta t \operatorname{Int}\left(\frac{t}{\Delta t}\right)
$$

is a time-periodic delay, and the function $\operatorname{Int}()$ rounds towards zero. The average delay, $\bar{\rho}$, is

$$
\bar{\rho}=\frac{1}{\Delta t} \int_{0}^{\Delta t} \rho(t) \mathrm{d} t=\frac{\Delta t}{2}
$$

Second, the quantization round off of $f$ means that the feedback forces are computed using integer multiples of the quantization step, $h$, and hence

$$
f\left(x\left(t_{j}\right)\right)=\operatorname{ph} \operatorname{Int}\left(\frac{x\left(t_{j}\right)}{h}\right),
$$

where we have assumed linear state feedback with control gain $p$. In making the above approximations, we assume a zero-order hold, namely, the force is kept constant over the interval $\Delta t$. A consequence of this assumption is that despite the periodically varying delay, the delayed feedback becomes a piecewise constant function.

\section{DISCRETE TIME: MICROCHAOTIC MAP}

Equation (3) with (4) and (6) leads to the governing equation

$$
\dot{x}(t)=q x(t)-p h \operatorname{Int}\left(\frac{1}{h} x\left(t-\Delta t \operatorname{Int}\left(\frac{1}{\Delta t} t\right)\right)\right)
$$

in continuous-time representation. The internal Int function refers to discretization in time and the external Int function represents quantization in space. Discretization in time is a linear effect that increases the dimension of the state space due to the inherent delay; quantization in space makes the problem strongly nonlinear. form

Equation (2) with (6) gives an equivalent semi-discrete

$$
\dot{x}(t)=q x(t)-p h \operatorname{Int}\left(\frac{x\left(t_{j}\right)}{h}\right), \quad t \in\left[t_{j}, t_{j+1}\right) .
$$

This equation can be solved over the interval $\left[t_{j}, t_{j+1}\right)$ to give the discrete governing equation in the form of the microchaos $\operatorname{map}^{7}$

$$
x\left(t_{j+1}\right)=a x\left(t_{j}\right)-b h \operatorname{Int}\left(\frac{x\left(t_{j}\right)}{h}\right),
$$

where

$$
a=\exp (q \Delta t)>1, \quad b=\frac{p}{q}(1-\exp (q \Delta t)) .
$$

The dynamics of (9) arise from the interplay between behaviors close to the trivial fixed point $x \equiv 0$ and those global to it. The local dynamics, when $|x|<h$, is governed by

$$
x\left(t_{j+1}\right)=a x\left(t_{j}\right) .
$$

Since $a>1$, the trivial fixed point is always locally "cyclic fold" unstable. The phrase "cyclic fold" means that the changes in $x$ occur monotonically. When $|x| \gg h$, then $b h \operatorname{Int}\left(\frac{x\left(t_{j}\right)}{h}\right)$ $\approx b x\left(t_{j}\right)$ and hence the global dynamics are governed by

$$
x\left(t_{j+1}\right)=(a-b) x\left(t_{j}\right) .
$$

With respect to the global dynamics, four different cases (labeled, respectively, A, B, C, and D) can be distinguished depending on the relation between $a$ and $b$. Local and global behaviors of the different cases are illustrated in Fig. 1. Different regions in the parameter plane $(a, b)$ are shown in Fig. 2.

In what follows, we show that three out of the four cases, namely, cases B, C, and D, present microchaos. The three conditions for chaos we use here are: ${ }^{17}$ (1) sensitive dependence on the initial conditions; (2) existence of closed invariant attractive sets; and (3) topological transitivity (mixing). First, the sensitive dependence on the initial conditions follows directly from the positiveness of the Lyapunov exponent $\Lambda=\ln (a)>0$ for all of the cases. Second, closed invariant attractive sets, which confine chaotic motions, are obtained. Third, the mixing property is not proven here, but we conjecture that existing methods based on the construction of an appropriate symbolic dynamics ${ }^{7,11}$ can be extended to establish topological transitivity of these maps.

\section{A. Case A: $a-b>1$}

The trivial fixed point is globally cyclic fold repelling [see Fig. 1(a)]. Since this case admits no sustained solution, we do not consider it further.

\section{B. Case B: $0<a-b<1$}

This case has been analyzed previously by Haller and Stepan. ${ }^{7}$ The neighborhood of the trivial fixed point is globally cyclic fold attracting [Fig. 1(c)]. Note that for sufficiently large $x_{j}$ ("large scale") the values of the iterates decrease [Fig. 1(c)]. 

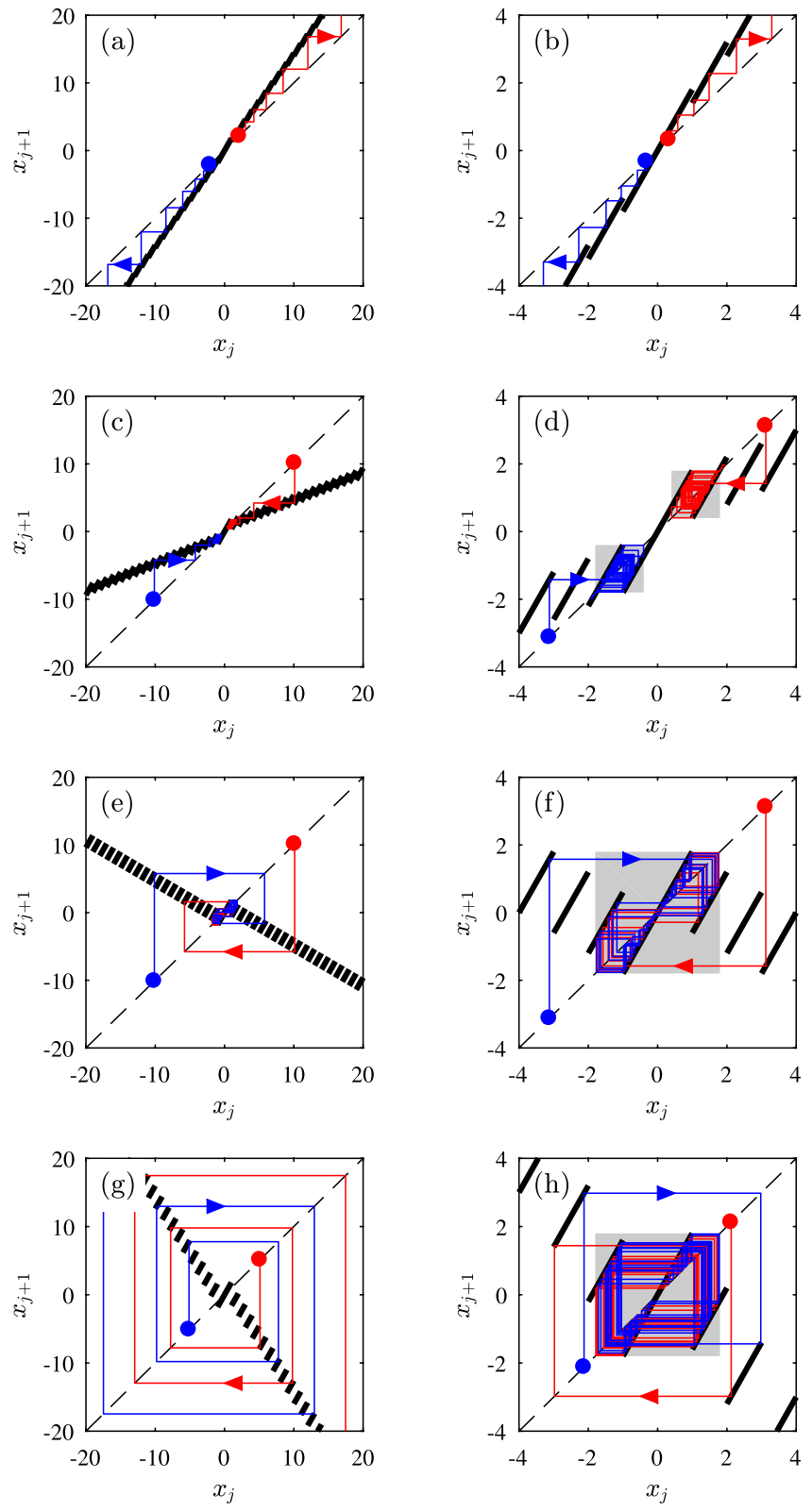

FIG. 1. Cobweb diagrams for (9) with $h=1$ when $a=1.8$ and [(a) and (b)] $b=0.4$, [(c) and (d)] $b=1.4,[(\mathrm{e})$ and (f) $b=2.4,[(\mathrm{~g})$ and $(\mathrm{h})] b=3.4$. The red and blue lines show different solutions associated with two different choices of the initial conditions denoted by red and blue dots, respectively. The thick black lines represent the right-hand side of (9). Left panels represent global dynamics, while right panels illustrate the dynamics closer to the trivial fixed point.

However, since the fixed point $x \equiv 0$ is unstable, the dynamics increase for small $x_{j}$ ("small scale") [Fig. 1(d)]. For certain parameter combinations, it can be proved that the dynamics are microchaotic. ${ }^{7}$ However, the numerical results in Fig. 2 suggest that microchaotic solutions exist at each point of the parameter region $0<a-b<1$. The closed invariant attractive set confining microchaotic solutions is

$$
\mathcal{A}_{\mathrm{B}+}=[n(a-b) h,(m(a-b)+a) h],
$$

for positive initial conditions ${ }^{7}$ and

$$
\mathcal{A}_{\mathrm{B}-}=[-(m(a-b)+a) h,-n(a-b) h],
$$

for negative initial conditions, where

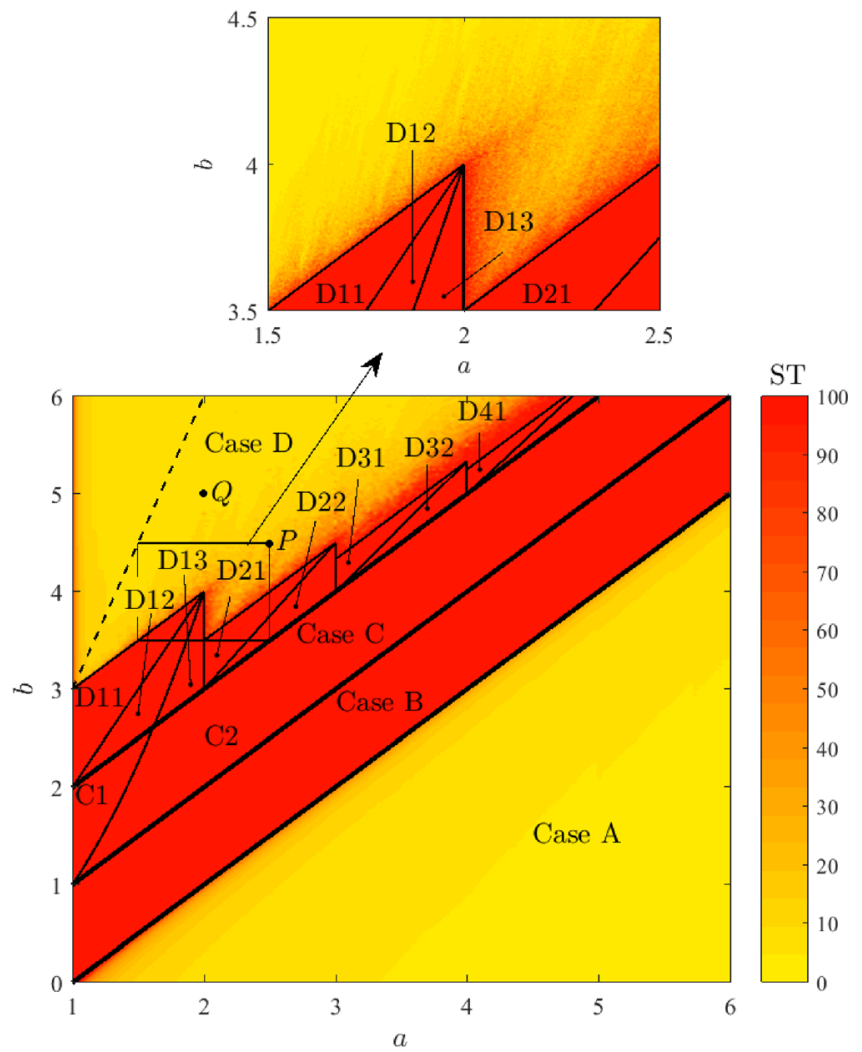

FIG. 2. Steady state behavior of (9). Yellow-red color code indicates the expected survival time (ST) determined via a series of numerical simulations. Parameter regions for cases A, B, C, and D are separated by thick lines, while individual sub regions associated with different sizes of domains of attractions are indicated by thin lines. The dashed line is $b=3 a$ (see text for discussion).

$$
n=\text { floor }\left(\frac{b}{(b+1-a) a}\right), \quad m=\operatorname{ceil}\left(\frac{(a-1) a-b}{(b+1-a) a}\right) .
$$

Note that the size of the invariant attractive set is scaled to the quantization step $h$. That is, for small quantization step, the amplitude of the chaotic motions is small. The fact that the chaotic dynamics are confined to a small region near the trivial fixed point justifies the term microchaos. The domain of attraction of $\mathcal{A}_{\mathrm{B}+}$ is $\mathcal{D}_{\mathrm{B}+}=(0, \infty)$, while the domain of attraction of $\mathcal{A}_{\mathrm{B}-}$ is $\mathcal{D}_{\mathrm{B}-}=(-\infty, 0){ }^{7}$

\section{Case C: $-1<a-b<0$}

The neighborhood of the trivial fixed point is globally period doubling attracting [Fig. 1(e)]. The phrase "period doubling" means that the changes in $x$ occur with alternating sign. Although this particular case has not been analyzed previously, numerical simulations indicate that these solutions are microchaotic. The closed invariant attractive set for case $\mathrm{C}$ is

$$
\mathcal{A}_{\mathrm{C} 1}=[-a h, a h] \backslash\left[\left(a^{2}-b\right) h,\left(b-a^{2}\right) h\right],
$$

when $b>a^{2}$ (see region C1 in Fig. 2) and

$$
\mathcal{A}_{\mathrm{C} 2}=[-a h, a h],
$$


when $b \leq a^{2}$ (see region $\mathrm{C} 2$ in Fig. 2). For details on the calculation of $\mathcal{A}_{\mathrm{C} 1}$ and $\mathcal{A}_{\mathrm{C} 2}$, see Appendix A. Similarly to case $\mathrm{B}$, the size of the invariant attractive set has been scaled to the quantization step $h$. The domain of attraction of $\mathcal{A}_{\mathrm{C} 1}$ and $\mathcal{A}_{\mathrm{C} 2}$ is $\mathcal{D}_{\mathrm{C}}=(-\infty, \infty)$.

\section{Case D: $a-b<-1$}

The neighborhood of the trivial fixed point is globally period doubling repelling [Fig. 1(g)]. This case has not been studied previously. Despite the fact that the fixed point is both locally and globally repelling, it is possible that a stable microchaotic solution exists [Fig. 1(h)]. Numerical simulations show that the regions D11, D12, D13, D21, D22, etc., in Fig. 2 are associated with permanent chaos. The boundaries of these parameter domains and the corresponding closed invariant attractive sets can be given as

$$
\begin{aligned}
& \text { D11: } 1<a<2 \text { and } 2 a<b<a+2, \\
& \mathcal{A}_{\mathrm{D} 11}= {[(a-b) h,(b-a) h] } \\
& \backslash[(a(b-a)-b) h,(b-a(b-a)) h],
\end{aligned}
$$

D12: $1<a<2$ and $a^{2}<b<2 a$ and $a+1<b$, $\mathcal{A}_{\mathrm{D} 12}=[-a h, a h] \backslash\left[\left(a^{2}-b\right) h,\left(b-a^{2}\right) h\right]$,

D13: $1<a<2$ and $a+1<b<a^{2}$,

$$
\mathcal{A}_{\mathrm{D} 13}=[-a h, a h] \text {, }
$$

D21: $2<a<3$ and $\frac{3}{2} a<b<a+\frac{3}{2}$, $\mathcal{A}_{\mathrm{D} 21}=[2(a-b) h, 2(b-a) h]$,

D22 : $2<a<3$ and $a+1<b<\frac{3}{2} a$, $\mathcal{A}_{\mathrm{D} 22}=[-a h, a h]$,

$$
\text { D31 : } 3<a<4 \text { and } \frac{4}{3} a<b<a+\frac{4}{3} \text {, }
$$$$
\mathcal{A}_{\mathrm{D} 31}=[3(a-b) h, 3(b-a) h],
$$

D32 : $3<a<4$ and $a+1<b<\frac{4}{3} a$,

$$
\mathcal{A}_{\mathrm{D} 32}=[-a h, a h] \text {, }
$$$$
\ldots
$$

(see Appendix A for details). Similar to cases B and C, the size of the invariant attractive set is scaled to the quantization step $h$. As opposed to cases B and C, the domain of attraction of the above attractive sets is not infinite, since the global dynamics (for large $|x|$ ) in case D is repelling. This means that for small quantization step, the solution may easily escape from the invariant attractive set due to large enough disturbances or do not even get inside this set if the initial condition is large enough [see the solutions in Figs. 1(c), 1(e), and 1(g) for cases B, C, and D, respectively].

\section{E. Transient microchaos}

In contrast to cases $\mathrm{B}$ and $\mathrm{C}$, transient microchaotic dynamics can also arise in case D. The term "transient microchaos" refers to metastable solutions that transiently survive close to the trivial equilibrium before diverging towards infinity. ${ }^{18,19}$ These transient solutions exist just above the regions D11, D21, D31, etc., and below the line $b=3 a$ indicated by dashed line in Fig. 2. Transient microchaotic dynamics can be characterized by the first passage or survival time (ST), namely, the time at which $|x|$ first exceeds a certain value, $x_{\mathrm{lim}}$. An added complexity is the fact that the ST is sensitive to the choice of initial condition (see Fig. 3). In general, the expected value of the ST cannot be determined analytically except for special cases (see Appendix B for the determination of the ST for point Q in Fig. 2).

In view of the above considerations, the ST in Fig. 2 was determined numerically as follows. Time history for a series of pairs $(a, b)$ was determined numerically for 20 different initial conditions distributed uniformly in the interval $[-h, h]$ with $h=1$ for $t_{\max }=100$ iteration steps and the time instant where $|x|$ first exceeded $x_{\text {lim }}=100$ was recorded and averaged. The color code indicates the averaged ST: yellow indicates $\mathrm{ST}=0$ and red indicates $\mathrm{ST}=\mathrm{t}_{\max }$.

It is observed that the ST in the region associated with case A is close to zero as expected, since both the openloop and the closed loop systems are unstable. The regions of case B and case C are both associated with red color. The ST in these regions is $t_{\max }=100$, which reflects the existence of long-lived bounded motions, namely, a permanent microchaos as shown in Figs. 1(d) and 1(f), respectively. For case D, there are choices of $(a, b)$ which are
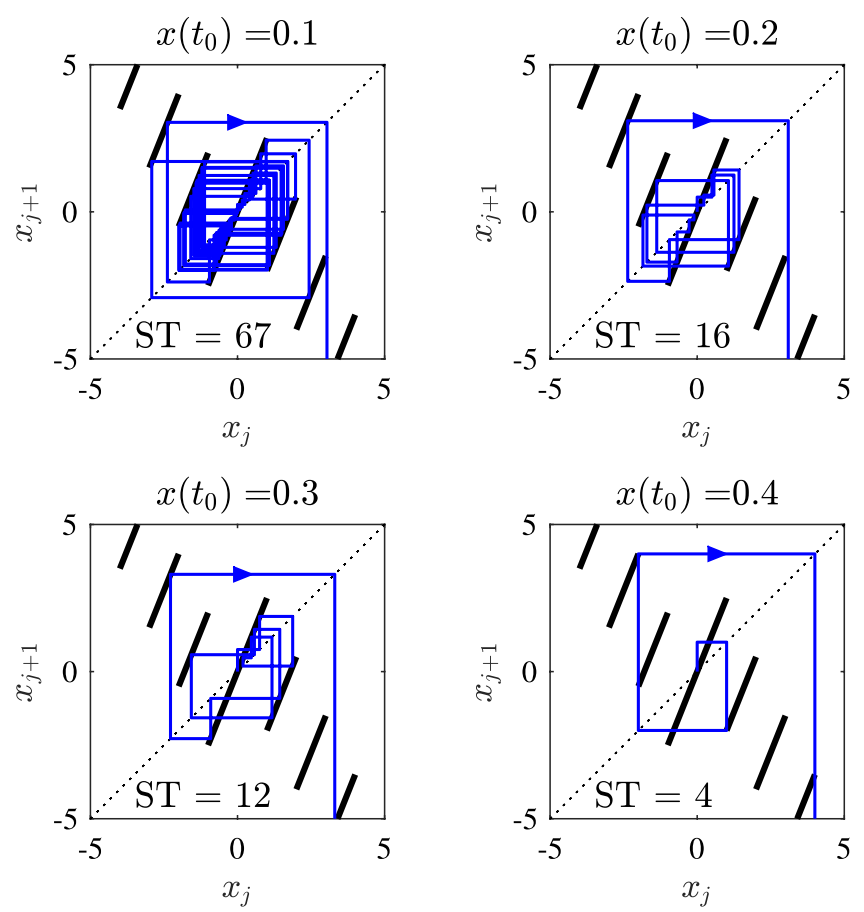

FIG. 3. Cobweb diagrams for case D with $h=1$ associated with point $\mathrm{P}$ $(a=2.5$ and $b=4.5)$ in Fig. 2 with different initial conditions $x\left(t_{0}\right)$. 
associated with long-lived bounded microchaos (red) as well as parameter choices with transient solutions (red-yellow scale).

\section{QUANTIZATION: HAYES EQUATION}

We anticipate that the behaviors exhibited by case D will have their counterpart in continuous time-delayed feedback control systems. Of particular interest is the possibility of stabilization through quantization of the feedback. To explore this possibility, we consider the Hayes equation ${ }^{16}$

$$
\dot{x}(t)=q x(t)-p x(t-T),
$$

where $q>0$ is the system parameter, $p$ is the control gain, and $T$ is the feedback delay. By rescaling time as $\tilde{t}:=q t$ and dropping the tilde immediately, we get

$$
\dot{x}(t)=x(t)-C x(t-\tau),
$$

where $\tau=T / q$ is the scaled feedback delay and $C=p / q>0$ is the scaled feedback gain. Biological applications of the Hayes equation arise in the description of the control of blood cell dynamics ${ }^{20,21}$ and the pupil light reflex. ${ }^{22}$ When $C=0$, the fixed point $x \equiv 0$ is an unstable node. When $\tau$ and $C$ are sufficiently large, stability is lost and we have an unstable spiral point. The stability boundaries of (26) in the plane $(C, \tau)$ are given by the line $C=1$ and the parametric curve

$$
C=\sqrt{\omega^{2}+1}, \quad \tau=\frac{1}{\omega} \operatorname{atan}(\omega),
$$

with $\omega \in[0, \infty)$. Quantization of (26) yields

$$
\dot{x}(t)=x(t)-C h \operatorname{Int}\left(\frac{x(t-\tau)}{h}\right),
$$

where $h$ is the quantization step. When $|x| \gg h$, then $C h \operatorname{Int}\left(\frac{x(t-\tau)}{h}\right) \approx C x(t-\tau)$ and hence the global dynamics are still governed by (26). Alternatively, one can write (28) in the form

$$
\dot{x}(t)= \begin{cases}\cdots & \\ x(t)-2 C h & \text { if } \quad 2 h \leq x(t-\tau)<3 h, \\ x(t)-C h & \text { if } \quad h \leq x(t-\tau)<2 h, \\ x(t) & \text { if }-h<x(t-\tau)<h, \\ x(t)+C h & \text { if }-2 h<x(t-\tau) \leq-h, \\ x(t)+2 C h & \text { if }-3 h<x(t-\tau) \leq-2 h, \\ \cdots & \end{cases}
$$

Figure 4 summarizes the behavior of (28) as a function of $C$ and $\tau$ when $h=1$. The semi-discretization numerical method was used to get the time history of the systems for different pairs of $(C, \tau)$ over the period $\left[0, t_{\max }\right]$ with $t_{\max }=100$. The initial condition for the simulations was $x(\theta) \equiv x_{\mathrm{IC}}, \theta \in[-\tau, 0]$, where $x_{\mathrm{IC}}$ is a constant chosen such that $x_{\mathrm{IC}} \leq h$. Solutions were declared to diverge if $|x(t)|$ $\geq x_{\lim }=100$ was satisfied. Yellow-red color code indicates the ST, i.e., the time when $|x(t)|$ exceeded $x_{\text {lim. }}$. It can be seen

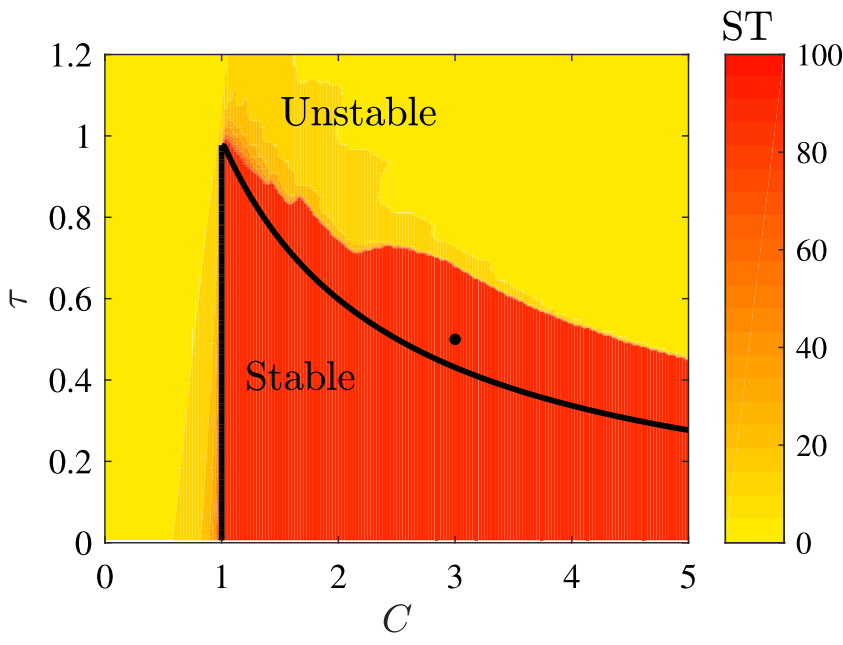

FIG. 4. Regions of steady state behaviors of Eqs. (26) and (28) when $h=1$. Solid lines indicate the stability boundaries for the Hayes equation (26). Yellow-red color code indicates the expected survival time (ST) for the quantized Hayes equation (26). The $\bullet$ indicates the point $C=3, \tau=0.5$ discussed in Fig. 5.

that bounded motions exists in the region where the Hayes equation (26) is unstable.

Figure 5 shows the effects of changing $h$ on the solutions of (28) when the parameters $C, \tau$ are chosen so that the fixed point of (26) is unstable (see $\bullet$ in Fig. 4). As can be seen, the amplitude of the oscillations can be reduced by decreasing $h$. However, for cases when $h<x_{\text {IC }}$, the solution gets out of the domain of attraction and its amplitude grows exponentially. Thus, there is a trade-off between $h$ and $x_{\text {IC }}$ (or between $h$ and the noise in the system). Decreasing $h$ may provide a solution which is a useful approximation for a stabilized fixed point for practical purposes (see the case $h=0.05$ in

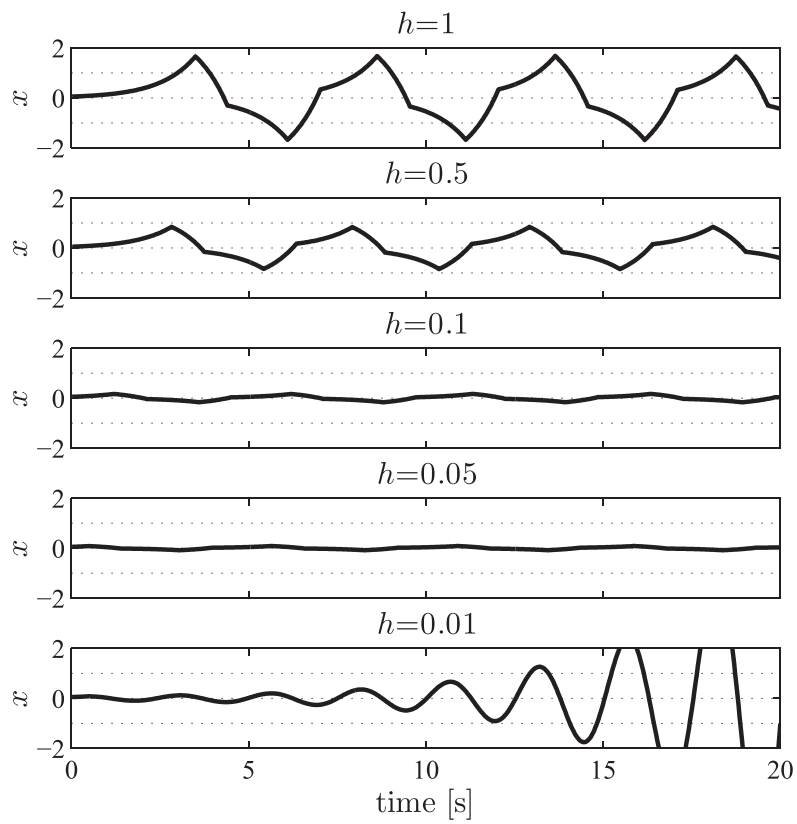

FIG. 5. Effect of quantization step size, $h$, on the solutions of (28) when $x_{\mathrm{IC}}=0.01, C=3$, and $\tau=0.5$. 
Fig. 5); however, further decrease of $h$ may destabilize the system.

\section{DISCUSSION}

Here, we have investigated the potential for "coarse grained" quantized feedback to stabilize an unstable feedback control system. We have shown that even when the fixed point is both locally and globally unstable feedback quantization can produce a long-lived bounded solution. This stabilized solution takes the form of an oscillatory fluctuation whose amplitude is proportional to the quantization step $h$. We did not directly address whether the combination of feedback quantization and time discretization could generate microchaotic solutions as proven for the closely related Eurich-Milton equation. ${ }^{11,37}$

One way that quantization can enter into the dynamics of time delayed feedback control is because of the presence of the sensory dead zones. For example, for stick balancing on the fingertip, there is a sensory dead zone of $1-3^{\circ}$ for the detection of vertical displacement angle in the anteriorposterior direction. ${ }^{15}$ Our observations suggests that the presence of this dead zone may not simply be a limitation for control but could be beneficial.

For the discrete-time system (9), the regions near the trivial fixed point where bounded solutions exist is located at higher gain values when $b>a+1$, while the system is unstable when $b<a-1$ (see Fig. 2). Similarly, for the quantized Hayes equation (28), bounded solutions exist at higher gain values when $C$ is larger than the stability boundary of the Hayes equation (26), while the system is unstable when $C<1$ (see Fig. 4). This shows that the beneficial effect of quantization can be utilized when the controller is tuned to "overreact" to the changes in the state variable.

Time discretization necessarily introduces a time delay into the dynamical system. This is because the state of the dynamical system at time $t \in\left[t_{j}, t_{j+1}\right], t_{j}=j \Delta t$ depends on its state at time $t_{j}$, where $\Delta t$ is the discretization step. Practical experience suggests that since the time discretization step can be made very small, it can be ignored; however, this is not always true. ${ }^{23}$ A major exception occurs when the feedback itself is time-delayed and quantized. ${ }^{7,11}$ Historically, the effect of piecewise constant, time-delayed feedback was extensively studied in early investigations into the dynamics of time-delayed feedback control. $^{24-30}$ Since experimental paradigms could be readily developed, it was possible to directly compare prediction with observation. ${ }^{30-33}$ It is important to note that in these models the feedback switching times could be precisely computed analytically and thus the solutions were obtained by piecing together exponential segments or spiral arcs. The use of numerical algorithms, such as the Euler-discretization, semi-discretization, or Runga-Kutta method introduces a low amplitude, microchaotic element to the dynamics. ${ }^{11}$ A related situation likely occurs in the use of computer algorithms to simulate the dynamics of integrate-and-fire neurons, ${ }^{34-36}$ although in this case, a formal demonstration that the dynamics are microchaotic has not yet been made.

Quantizing feedback is expected to be an effective stabilization strategy provided that (1) the fixed point of the uncontrolled dynamical system is exponentially unstable, (2) the fixed point in the presence of continuous delayed feedback is an unstable spiral point, and (3) the noise intensity is not too high. A consequence of time-delayed, quantized feedback is that both open-loop and closed-loop unstable fixed points are replaced by a stable oscillation. If, in addition, there is time discretization, then the oscillations will be replaced by a microchaotic fluctuation. If the amplitude of the generated oscillation is not too large, then the solution may be acceptable for certain applications. Indeed, the long lived balanced state obtained by experts who balance a stick on their fingertip is a transient microchaotic solution. ${ }^{15}$ Another application of these results is that the control gain can be increased by increasing the size of the quantization step, which can be a useful feature, for instance, in position control in the presence of dry friction.

\section{ACKNOWLEDGMENTS}

The research has received funding from the European Research Council under the European Union's Seventh Framework Programme (FP7/2007-2013)/ERC Advanced Grant Agreement No. 340889 (SG,IT) and from the William R. Kenan, Jr., Charitable trust (JM).

\section{APPENDIX A: CLOSED INVARIANT SETS FOR CASES $B, C$ AND D}

The closed invariant attractive sets are the sets of minimum size, where the solutions never escape from. These sets are determined by the parameters $a$ and $b$ and the quantization step $h$.

The closed invariant attractive sets for case $\mathrm{B}$, when $0<a-b<1$, were given by Haller and Stepan. ${ }^{7}$

For case $\mathrm{C}$, when $-1<a-b<0$, there are two different cases. If $a^{2}-b>0$, then it is easy to see that the solutions never leaves the set $\mathcal{A}_{\mathrm{C} 2}$ given in (17), see panel $\mathrm{C} 2$ in Fig. 6. However, when $a^{2}-b<0$, then the solutions never return to the set $\left[\left(a^{2}-b\right) h,\left(b-a^{2}\right) h\right]$ once they leaved it, hence the invariant attractive set is $\mathcal{A}_{\mathrm{C} 1}$ given in (16), see panel $\mathrm{C} 1$ in Fig. 6.

For case $\mathrm{D}$, when $a-b<-1$, there are several subcases. Consider first the case when $1<a<2$. If $a>b-a$ and $a^{2}-b>0$, then the invariant set is $\mathcal{A}_{\mathrm{D} 13}$ given by (20), see panel D13 in Fig. 6. If $a>b-a$ but $a^{2}-b<0$, then the solutions never return to the set $\left[\left(a^{2}-b\right) h,\left(b-a^{2}\right) h\right]$ once they leave it, hence the invariant set is $\mathcal{A}_{\mathrm{D} 12}$ given by (19). If $a<b-a$, then upper bound of the invariant set becomes $b-a$, the solutions never return to the set $[(a(b$ $-a)-b) h,(b-(a(b-a))) h]$ once they leave it, hence the invariant set is $\mathcal{A}_{\mathrm{D} 11}$ given by (18).

Consider now the case when $2<a<3$. If $a>2(b-a)$, then the invariant set is $\mathcal{A}_{\mathrm{D} 22}$ given by (22), see panel D22 in Fig. 6. If $a<2(b-a)$, then the invariant set is $\mathcal{A}_{\mathrm{D} 21}$ given by (21), see panel D21 in Fig. 6. 

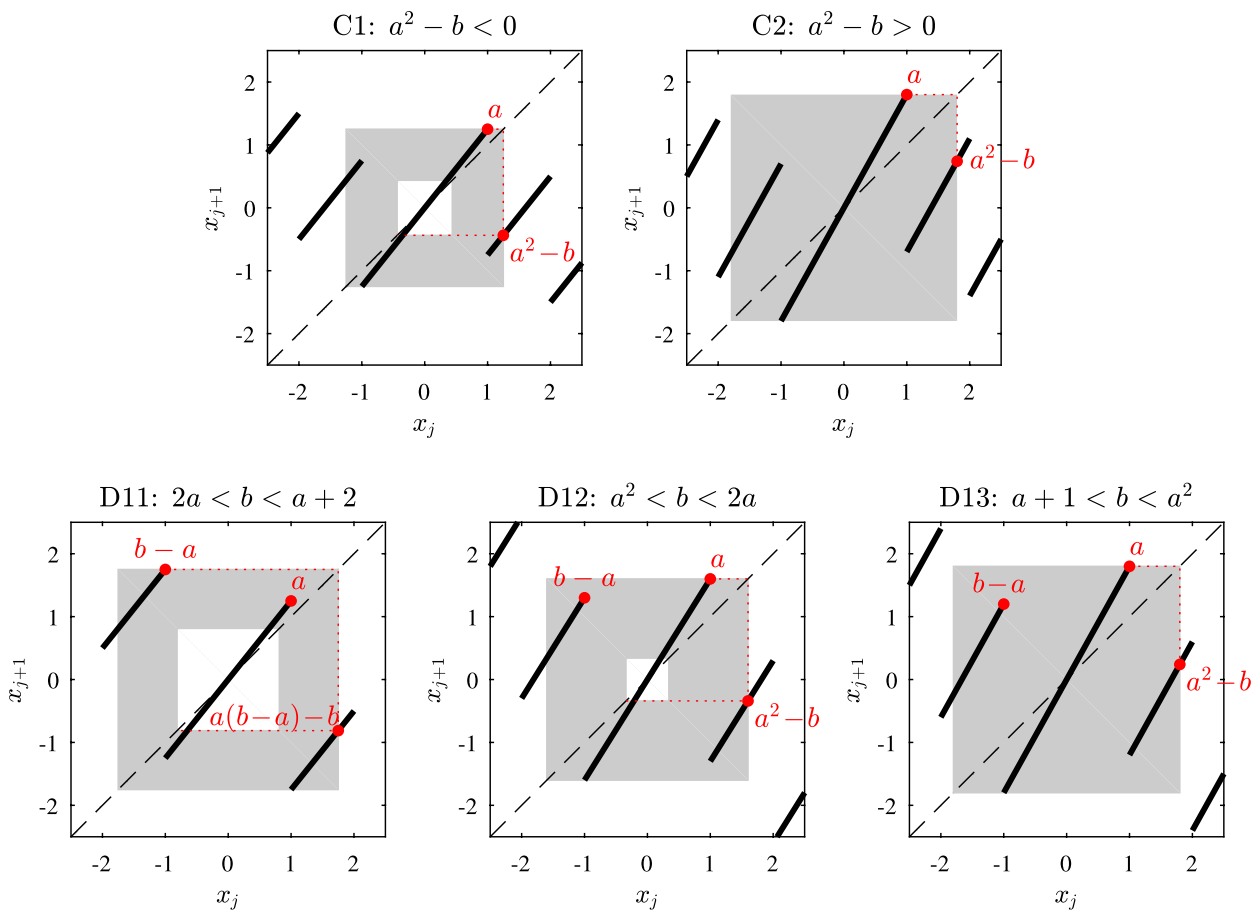

FIG. 6. Illustration of the invariant sets for different cases.
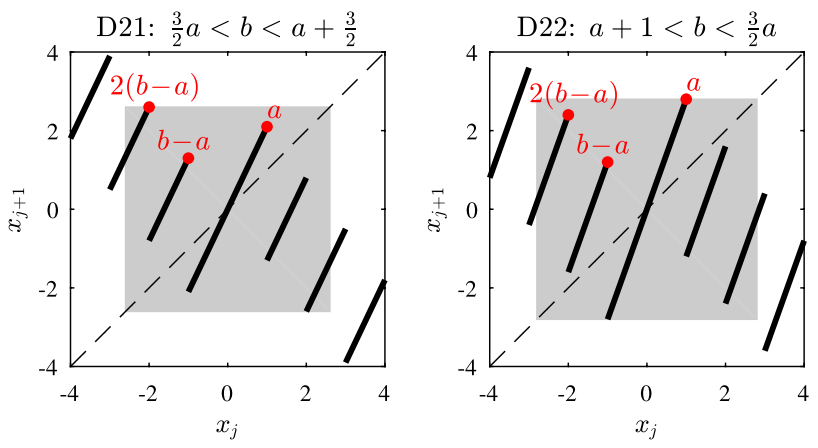

The invariant sets for the cases when $a>3$ can be obtained similarly

\section{APPENDIX B: SURVIVAL TIME CALCULATION}

The survival time (ST) for (9) with $a=2$ and $b=5$ is calculated. This parameter combination corresponds to point $\mathrm{Q}$ in Fig. 2. Without loss of generality, the quantization step can be set to $h=1$.

First, we determine the expected ST for the interval $I_{0}=[-2,2]$, i.e., the average time duration until the solutions initiated from the interval $I_{0}$ stay in $I_{0}$. For this calculation, $I_{0}$ is divided into subintervals, where the ST is invariant. It is easy to see, for instance, that a solution escapes from the interval $I_{0}$ after one step of iteration if $x\left(t_{0}\right) \in I_{1}$, where

$$
I_{1}=[-1.5,-1) \cup(1,1.5]
$$

The solutions escape after two iterations if $x\left(t_{0}\right) \in I_{2}$, where

$$
I_{2}=[-2,-1.75) \cup[-1,-0.5) \cup(0.5,1] \cup(1.75,2]
$$

is the pre-image of $I_{1}$. The solution escapes after three iterations if $x\left(t_{0}\right) \in I_{3}$, where

$$
\begin{gathered}
I_{3}=[-1.625,-1.5) \cup[-1,-0.875) \cup[-0.375,-0.25) \\
\cup(0.25,-0.375] \cup(0.875,1] \cup(1.5,1.625]
\end{gathered}
$$

is the pre-image of $I_{2}$. By continuing the pre-image mapping, an infinite series of subintervals $I_{k}, k=1,2, \ldots$ is obtained, each associated with a ST equal to $k$ (see Fig. 7). The length of the subintervals is

$$
\ell\left(I_{k}\right)=\frac{2 k}{2^{k}} \quad k=1,2, \ldots
$$

The length of the union of these subintervals is

$$
\sum_{k=1}^{\infty} \ell\left(I_{k}\right)=\sum_{k=1}^{\infty} \frac{2 k}{2^{k}}=\sum_{k=1}^{\infty} k\left(\frac{1}{2}\right)^{k-1},
$$

which can be calculated as follows. Introduce the function

$$
f(q):=\sum_{k=1}^{\infty} q^{k}=\frac{q}{1-q},
$$

where $0<q<1$. The derivative of $f$ with respect to $q$ is 

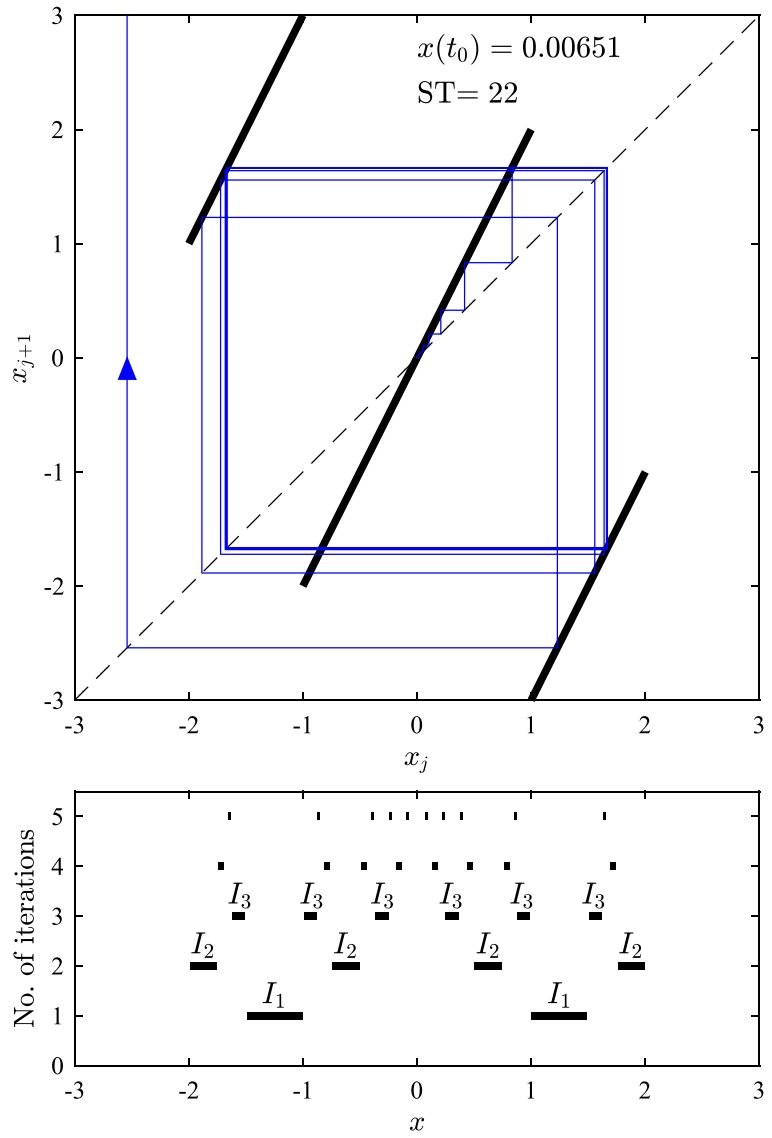

FIG. 7. Cobweb diagram for case D with $h=1$ associated with point Q $(a=2$ and $b=5)$ in Fig. 2 and the structure of the associated subintervals $I_{j}$, $j=1,2, \ldots$.

$$
f^{\prime}(q)=\sum_{k=1}^{\infty} k q^{k-1}=\frac{(1-q)+q}{(1-q)^{2}}=\frac{1}{(1-q)^{2}} .
$$

The infinite sum in (B5) can be given by setting $q=1 / 2$ in (B7) as

$$
\sum_{k=1}^{\infty} \ell\left(I_{k}\right)=f^{\prime}\left(\frac{1}{2}\right)=\frac{1}{\left(1-\frac{1}{2}\right)^{2}}=4 .
$$

Thus, the union of the subintervals $I_{1}, I_{2}, \ldots$ covers the whole interval $I_{0}$.

The average $\mathrm{ST}$ for $I_{0}$ can be given as

$$
\mathrm{ST}_{I_{0}}=\frac{1}{\ell\left(I_{0}\right)} \sum_{k=1}^{\infty} k \frac{2 k}{2^{k}}=\frac{1}{\ell\left(I_{0}\right)} \sum_{k=1}^{\infty} k^{2}\left(\frac{1}{2}\right)^{k-1} .
$$

Now introduce the function

$$
g(q):=\sum_{k=1}^{\infty} k q^{k}=q \sum_{k=1}^{\infty} k q^{k-1}=q f^{\prime}(q)=\frac{q}{(1-q)^{2}},
$$

where $0<q<1$. The derivative of $g$ with respect to $q$ is

$$
g^{\prime}(q)=\sum_{k=1}^{\infty} k^{2} q^{k-1}=\frac{(1-q)^{2}+2 q(1-q)}{(1-q)^{4}}=\frac{1-q^{2}}{(1-q)^{4}} .
$$

The infinite sum in (B9) can be given by setting $q=1 / 2$ in (B11) as

$$
\mathrm{ST}_{I_{0}}=\frac{1}{\ell\left(I_{0}\right)} g^{\prime}\left(\frac{1}{2}\right)=\frac{1}{4} \frac{1-\left(\frac{1}{2}\right)^{2}}{\left(1-\frac{1}{2}\right)^{4}}=\frac{12}{4}=3 .
$$

This is the average ST for the solutions initiated from the interval $I_{0}$. Figure 7 shows a sample solution for a particular initial condition $x\left(t_{0}\right)=0.00651$ with $\mathrm{ST}=22$.

${ }^{1}$ A. Kubanek, M. Koch, C. Sames, A. Ourjoumtsev, P. W. H. Pinkse, and G. Rempe, "Photon-by-photon feedback control of a single atom trajectory," Nature 462, 898-901 (2009).

${ }^{2}$ M. L. Homer, A. V. Nurmikko, J. P. Donoghue, and L. R. Hochberg, "Implants and decoding for intracortical brain computer interfaces," Annu. Rev. Biomed. Eng. 15, 383-405 (2013).

${ }^{3}$ D. B. Keenan, J. J. Mastrototaro, G. Voskanyan, and G. M. Steil, "Delays in minimally invasive continuous glucose monitoring device: A review of current technology,” J. Diabetes Sci. Technol. 3, 1207-1214 (2009).

${ }^{4} \mathrm{~J}$. I. Ge and G. Orosz, "Optimal control of connected vehicle systems with communication delay and driver reaction time," IEEE Trans. Intell. Transp. Syst. 18(8), 2056-2070 (2017).

${ }^{5} \mathrm{O}$. Feely, "A tutorial introduction to non-linear dynamics and chaos and their application to sigma-delta modulators," Int. J. Circ. Theor. Appl. 25, 347-367 (1997).

${ }^{6} \mathrm{~J}$. Reiss, "Understanding sigma-delta modulation: The solved and unsolved issues," J. Audio Eng. Soc. 56, 49-64 (2008); available at http:// www.aes.org/e-lib/browse.cfm?elib=14375.

${ }^{7}$ G. Haller and G. Stepan, "Micro-chaos in digital control," J. Nonlinear Sci. 6, 415-448 (1996).

${ }^{8}$ E. Enikov and G. Stepan, "Microchaotic motion of digitally controlled machines," J. Vib. Control 4, 427-443 (1998).

${ }^{9}$ P. Murphy, M. Xie, Y. Li, M. Ferdowsi, N. Patel, F. Fatehi, A. Homaifar, and F. Lee, "Study of digital vs analog control," in Power Electronics Seminars Proceedings (Center for Power Electronics Systems (CPES), 2002), pp. 203-206.

${ }^{10}$ G. F. Franklin, J. D. Powell, and A. Emami-Naeini, Feedback Control of Dynamic Systems, 6th ed. (Prentice Hall, New York, 2010).

${ }^{11}$ T. Insperger, J. Milton, and G. Stepan, "Semidiscretization for timedelayed neural balance control," SIAM J. Appl. Dyn. Sci. 14, 1258-1277 (2015).

${ }^{12}$ B. A. Kovacs and T. Insperger, "Retarded, neutral and advanced differential equation models for balancing using an accelerometer," Int. J. Dynam. Control (published online 2017).

${ }^{13} \mathrm{C}$. von Hofsten, "Structuring of early reaching movements: A longitudinal study," J. Motor Behav. 23, 280-292 (1991).

${ }^{14}$ T. E. Milner and M. M. Ijaz, "The effect of accuracy constraints in threedimensional movement kinematics," Neuroscience 35, 365-374 (1990).

${ }^{15}$ J. Milton, R. Meyer, M. Zhvanetsky, S. Ridge, and T. Insperger, "Control at stability's edge minimizes energetic costs: Expert stick balancing," J. R. Soc. Interface 13, 20160212 (2016).

${ }^{16} \mathrm{~N}$. D. Hayes, "Roots of the transcendental equation associated with a certain differential-difference equation," J. London Math. Soc. 25, 226-232 (1950).

${ }^{17}$ S. Wiggins, Chaotic Transport in Dynamical Systems (Springer-Verlag, New York, 1992).

${ }^{18}$ G. Csernak and G. Stepan, "Life expectancy of transient microchaotic behavior," J. Nonlinear Sci. 15, 63-91 (2005).

${ }^{19}$ Y.-C. Lai and T. Tel, Transient Chaos: Complex Dynamics on Finite Time Scales (Springer, New York, 2011).

${ }^{20} \mathrm{M}$. C. Mackey and L. Glass, "Oscillation and chaos in physiological control systems," Science 197, 287-289 (1977).

${ }^{21}$ M. C. Mackey, "Periodic auto-immune hemolytic anemia: An induced dynamical disease," Bull. Math. Biol. 41, 829-834 (1979).

${ }^{22}$ A. Longtin and J. G. Milton, "Modelling autonomous oscillations in the human pupil light reflex using nonlinear delay-differential equations," Bull. Math. Biol. 51, 605-624 (1989).

${ }^{23}$ Y. Kuang, Delay Differential Equations With Application in Population Dynamics (Academic Press, New York, 1993).

${ }^{24} \mathrm{U}$. an der Heiden and M. C. Mackey, "The dynamics of production and destruction: Analytic insight into complex behavior," J. Math. Biol. 16, 75-101 (1982). 
${ }^{25} \mathrm{U}$. an der Heiden and M. C. Mackey, "Mixed feedback: A paradigm for regular and irregular oscillations," in Temporal Disorder in Human Oscillatory Systems (Springer-Verlag, New York, 1987), pp. 30-46.

${ }^{26}$ U. an der Heiden, A. Longtin, M. C. Mackey, J. G. Milton, and R. Scholl, "Oscillatory modes in a nonlinear second order differential equation with delay,” J. Dyn. Diff. Eqn. 2, 423-449 (1990).

${ }^{27}$ W. Bayer and U. an der Heiden, "Oscillatory types and bifurcations of a nonlinear second-order differential-difference equation," J. Dyn. Diff. Eqn. 10, 303-326 (1998).

${ }^{28}$ W. Bayer and U. an der Heiden, "Delay-differential equation with discrete feedback: Explicit formulae for infinitely many co-existing solutions," J. Appl. Math. Mech. 87, 471-479 (2007).

${ }^{29}$ J. Bélair and J. G. Milton, "Itinerary of a discontinuous map from the continued fraction expansion," Appl. Math. Lett. 1, 339-342 (1988).

${ }^{30}$ J. G. Milton and A. Longtin, "Evaluation of pupil constriction and dilation from pupil cycling measurements," Vision Res. 30, 515-525 (1990).
${ }^{31}$ A. Longtin and J. G. Milton, "Complex oscillations in the human pupil light reflex with 'mixed' and delayed feedback," Math. Biosci. 90, 183-199 (1988).

${ }^{32}$ A. Longtin, J. G. Milton, J. E. Bos, and M. C. Mackey, "Noise and critical behavior of the pupil light reflex at oscillation onset," Phys. Rev. A 41, 6992-7005 (1990).

${ }^{33}$ J. Losson, M. C. Mackey, and A. Longtin, "Solution multistability in first order nonlinear differential delay equations," Chaos 3, 167-176 (1993).

${ }^{34}$ D. Hansel, G. Mato, C. Meunier, and L. Neltner, "On numerical simulations of integrate-and-fire neural networks," Neural Comput. 10, 467-483 (1998).

${ }^{35}$ B. F. Nielsen and J. Wyller, "III-posed point neuron models," J. Math. Neurosci. 6, 7 (2016).

${ }^{36} \mathrm{M}$. J. Shelley and L. Tao, "Efficient and accurate time-stepping schemes for integrate-and-fire neural networks," J. Comput. Neurosci. 11, 111-119 (2001).

${ }^{37}$ C. W. Eurich and J. G. Milton, "Noise-induced transitions in human postural sway,” Phys. Rev. E 54, 6681-6684 (1996). 